\title{
Anlotinib as a molecular targeted therapy for tumors (Review)
}

\author{
YI GAO $^{1-3^{*}}$, PENGFEI LIU ${ }^{2,4^{*}}$ and RUIHUA SHI ${ }^{1,5}$ \\ ${ }^{1}$ School of Medicine, Southeast University, Nanjing, Jiangsu 210009; ${ }^{2}$ Department of Gastroenterology, \\ The Jiangyin Clinical College of Xuzhou Medical University, Jiangyin, Jiangsu 214400; \\ ${ }^{3}$ Jiangsu Provincial Key Laboratory of Radiation Medicine and Protection, School of Radiation Medicine and Protection, \\ Medical College of Soochow University; ${ }^{4}$ State Key Laboratory of Radiation Medicine and Protection, \\ Medical College of Soochow University, Suzhou, Jiangsu 215123; ${ }^{5}$ Department of Gastroenterology, Zhongda Hospital, \\ Affiliated Hospital of Southeast University, Nanjing, Jiangsu 210009, P.R. China
}

Received December 20, 2019; Accepted April 30, 2020

DOI: $10.3892 / \mathrm{ol} .2020 .11685$

\begin{abstract}
Angiogenesis has an essential role in tumor growth and metastasis, and blocking this pathway has been a successfully utilized strategy in the clinical treatment of cancer. Anlotinib (AL3818) is a novel oral receptor tyrosine kinase inhibitor targeting vascular endothelial growth factor receptor 2 and 3, fibroblast growth factor 1-4, platelet-derived growth factor receptor $\alpha$ and $\beta, c-K i t$ and Ret. Anlotinib exerts inhibitory effects on tumor growth and angiogenesis and received its first approval as a third-line treatment for refractory advanced non-small-cell lung cancer in May 2018 and its second approval as a second-line treatment for advanced soft-tissue sarcoma in June 2019 in the People's Republic of China. Anlotinib has encouraging efficacy and a manageable and tolerable safety profile in a broad range of malignancies, including medullary thyroid cancer, renal cell cancer, gastric cancer and esophageal squamous cell carcinoma. In the present review, the preclinical and clinical trials of anlotinib were summarized with a focus on safety evaluation and adverse event management.
\end{abstract}

\section{Contents}

1. Introduction

2. Preclinical studies of anlotinib

3. Clinical trials of anlotinib

Correspondence to: Professor Ruihua Shi, Department of Gastroenterology, Zhongda Hospital, Affiliated Hospital of Southeast University, 87 Dingjiaqiao, Nanjing, Jiangsu 210009, P.R. China

E-mail: ruihuashi@126.com

${ }^{*}$ Contributed equally

Key words: anlotinib, receptor tyrosine kinase inhibitor, vascular endothelial growth factor receptor, clinical trial, safety management
4. Summary of AEs associated with anlotinib in the treatment of advanced cancers

5. Management of drug-associated AEs

6. Future directions

7. Conclusion

\section{Introduction}

Cancer has always been a major public health problem worldwide, with 18.1 million new cases and 9.6 million deaths estimated in 2018 (1). Despite continuous advances in multidisciplinary regimens, including surgery, chemotherapy and radiotherapy, cancer still has an unfavorable prognosis. Conventional cytotoxic chemotherapy is the cornerstone of antitumor treatment, particularly for advanced cancer. The development of systemic toxicity and drug resistance to chemotherapy emphasizes the pressing need for better therapeutic strategies to prolong the lifespan of patients. In recent years, with the rapid progression of precision medicine, an increasing number of scientists have focused on developing precise therapeutic approaches by identifying novel molecular targeted therapies for the treatment of various types of cancer.

Angiogenesis has an essential role in tumor growth and metastasis, and consequently, blocking this pathway has been a successfully utilized strategy in the clinical treatment of cancer (2,3). Receptor tyrosine kinases (RTKs) are among the most promising therapeutic targets that modulate cell proliferation, growth, angiogenesis and metastasis in a wide range of cancers, including non-small-cell lung cancer (NSCLC), colorectal cancer (CRC) and breast cancer (4-8). The RTK family includes insulin receptors and numerous growth factor receptors, including epidermal growth factor receptor (EGFR), platelet-derived growth factor receptor (PDGFR), vascular endothelial growth factor receptor (VEGFR), nerve growth factor receptor (NGFR) and fibroblast growth factor receptor (FGFR), among others (9). In recent decades, there have been unprecedented advances in the development of various RTK inhibitors (10-15). Several targeted drugs, including gefitinib, erlotinib and crizotinib, have been approved by the Food and Drug Administration of U.S.A. for NSCLC treatment. Of note, 
anti-angiogenesis drugs, including bevacizumab and recombinant human endostatin, have become important treatment options for NSCLC $(16,17)$. However, no standard third-line treatment for NSCLC is available in China for patients who progressed after second-line chemotherapy. Considering the limited therapeutic response, there is still a requirement to overcome drug resistance and achieve target-specific sensitivity to decrease off-target-mediated toxicity.

Anlotinib (AL3818) is a novel oral RTK inhibitor targeting VEGFR-2 and -3, FGFR1-4, PDGFR- $\alpha$ and $-\beta$, c-Kit and Ret (18), thereby exerting inhibitory effects on tumor growth and angiogenesis, as illustrated in the schematic in Fig. 1. Anlotinib was approved and launched in the People's Republic of China in May 2018 as a third-line treatment for patients with refractory advanced NSCLC after $\geq 2$ lines of systemic chemotherapy and it received its second approval in June 2019 as a second-line treatment for patients with advanced alveolar soft-part sarcoma (ASPS), clear-cell sarcoma and other types of advanced soft tissue sarcoma (STS) after one line of a chemotherapy regimen containing anthracycline. In addition, several phase II/III clinical trials of anlotinib have been completed or are ongoing for various cancer types, including STS, renal cell carcinoma (RCC), hepatocellular carcinoma, ovarian cancer, esophageal squamous cell carcinoma (ESCC), gastric cancer, CRC, nasopharyngeal carcinoma and thyroid carcinoma. In the present review, the preclinical data and clinical trials of anlotinib are summarized and, more importantly, the safety evaluation and management of adverse events associated with the clinical use of anlotinib for cancer treatment are fully described.

\section{Preclinical studies on anlotinib}

A variety of basic studies have been dedicated to assessing the antitumor effect of anlotinib in vitro and in vivo. In vitro, anlotinib inhibits cell viability and induces apoptosis in human lung cancer cells, which in turn enhances the cytotoxicity of anlotinib and amplifies its anti-angiogenic effect through JAK2/STAT3/VEGFA signaling (19). Anlotinib exerts anti-tumor effects by inhibiting osteosarcoma cell growth, metastasis and angiogenesis through dual blockade of the VEGFR2 and MET signaling pathways (20). Anlotinib also exhibits promising anti-tumor effects on papillary thyroid cancer and anaplastic thyroid cancer cell lines (21). Lin et al (22) demonstrated that anlotinib inhibited angiogenesis by suppressing the activation of VEGFR2, PDGFR $\beta$ and FGFR1, as well as downstream ERK signaling. Furthermore, anlotinib has a better antiangiogenic effect than sunitinib, sorafenib and nintedanib when administered at the same concentration (22). Encouraged by its promising inhibitory effect on several cancer cell lines, studies further explored the potential anti-tumor activity of anlotinib in vivo. The results revealed that anlotinib had a potent inhibitory effect in human xenograft models of multiple cancer types when administered alone or in combination with chemotherapy (19-21,23).

Chemotherapy is a potent treatment option for cancer patients. The emergence of multidrug resistance is one of the major reasons for local recurrence or treatment failure in patients. In vitro, Wang et al (20) indicated that anlotinib increased the inhibitory effect of cisplatin (DDP) on osteosarcoma cell proliferation and promoted DDP-induced apoptosis when administered at a low concentration $(1 \mu \mathrm{M})$. In vivo, treatment with the combination of anlotinib and DDP significantly decreased tumor weight and volume compared with anlotinib or DDP alone (20). The above data indicated that anlotinib increases the sensitivity of osteosarcoma cells to DDP in vitro and in vivo. It has been acknowledged that activation of the FGFR signaling pathway promotes chemotherapy resistance (24-27). Anlotinib exerts its inhibitory effects by suppressing several targets, including FGFR1-4. Thus, anlotinib may be useful in enhancing the response to chemotherapy. Furthermore, its broad target range may also contribute to overcoming drug resistance induced by previous chemo- or targeted therapies.

However, anlotinib inevitably faces the problem of drug resistance at the late stage of cancer treatment. MicroRNAs (miRs) have a dual role in promoting drug sensitivity and inducing drug resistance in cancer treatment $(28,29)$. A study by Wang et al (30) suggested that silencing the expression of the gene metastasis-associated in colon cancer-1 via miR-940 increased the antitumor effect of anlotinib on the proliferation and invasive growth of CRC cells. Ma et al (31) reported that miR-6077 inhibited the expression of glucose transporter 1 and enhanced the anti-tumor effect of anlotinib on patient-derived lung adenocarcinoma cells. Another study indicated that miR-596 promoted the sensitivity of osteosarcoma to anlotinib by targeting survivin (32). Furthermore, Lu et al (33) revealed that supplementing exogenous $\mathrm{C}-\mathrm{X}-\mathrm{C}$ chemokine ligand 2 (CXCL2) may be a possible mechanism by which to circumvent anlotinib resistance. Despite the continuous efforts made by researchers in recent years, available approaches to overcoming drug resistance are still limited and have not been applied in the clinical setting.

\section{Clinical trials of anlotinib}

The efficacy of anlotinib in the treatment of various solid tumors has been assessed in several phase I, II and III studies (summarized in Table I).

Phase I. A phase I clinical trial was performed by Sun et al (34) to evaluate the pharmacokinetic variables, safety profile and anti-tumor properties of anlotinib in patients with advanced refractory solid malignancies and to establish the recommended phase II dose and schedule. A total of 35 patients were enrolled in this study, including two cohorts of patients who received anlotinib at doses of 5 or $10 \mathrm{mg}$ per person/day on the 4/0 schedule (consecutively for 28 days) and three cohorts of patients who received anlotinib at doses of 10,12 or $16 \mathrm{mg}$ per person/day on the $2 / 1$ schedule ( 2 weeks on/1 week off) for two cycles. Dose-limiting toxicity (DLT) was grade 3 hypertension at $10 \mathrm{mg}$ on the $4 / 0$ schedule. On the $2 / 1$ schedule, DLT was grade 3 hypertension or grade 3 fatigue at $16 \mathrm{mg}$. The pharmacokinetic assessment demonstrated that anlotinib was rapidly absorbed through the intestine and had a long elimination half-life of $96 \mathrm{~h}$, which resulted in significant accumulation in the plasma during multiple oral doses. The maximum tolerated dose was determined to be $12 \mathrm{mg} /$ day. The recommended dosage regimen for the phase II trial was $12 \mathrm{mg} /$ day on the $2 / 1$ schedule. The antitumor response of anlotinib was assessed in 


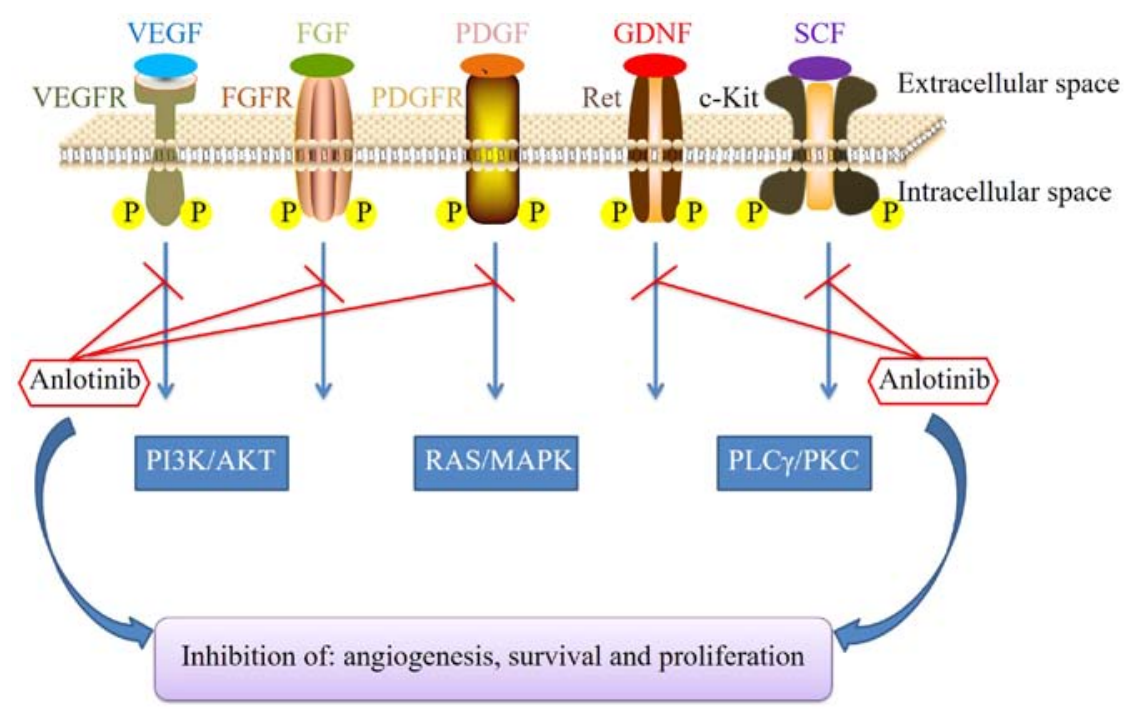

Figure 1.Mechanism of action of anlotinib.FGFR, fibroblast growth factor receptor; GDNF, glial cell line-derived neurotrophic factor; MAPK, mitogen-activated protein kinase; PDGFR, platelet-derived growth factor receptor; PLC $\gamma$, phospholipase $\gamma 1$; PKC, protein kinase C; RAS, rat sarcoma protein; SCF, stem-cell factor; VEGFR, vascular endothelial growth factor receptor; P, phosphate.

20 of the 21 patients who received $12 \mathrm{mg} /$ day anlotinib on the $2 / 1$ schedule. Partial response was noted in 3 patients $(15 \%)$ and stable disease was noted in 14 patients $(70 \%)$, including 12 patients with tumor burden shrinkage and 3 patients $(15 \%)$ with progressive disease. The types of cancer that responded to anlotinib included colon adenocarcinoma, NSCLC, renal clear cell cancer, medullary thyroid carcinoma (MTC), STS, melanoma, thymic carcinoma and adenoid cystic carcinoma. The most frequent serious adverse events (AEs) reported were hypertension, triglyceride elevation, hand-foot skin reaction (HFSR) and lipase elevation. In this phase I study, the toxicity of anlotinib was indicated to be manageable and the recommended regimen was well tolerated.

Another phase Ib study was performed at a single US center with subjects with ovarian, cervical and endometrial cancers (grant no. NCT02558348) (35). The author also recommended $12 \mathrm{mg}$ daily with a 2-week on/1-week off regimen.

\section{Phase II}

Anlotinib for advanced NSCLC and SCLC. Lung cancer remains the most common type of malignancy worldwide, with 2.1 million new lung cancer cases and 1.8 million deaths estimated in 2018 (1). NSCLC and SCLC are the two primary types of lung cancer. NSCLC accounts for $80-85 \%$ of diagnosed lung cancers, whereas SCLC accounts for $\sim 15 \%$ of cases (36). Most people are diagnosed after the cancer has already spread and only a minority of patients may be treated by surgery. Current treatment options for advanced lung cancer remain limited, and the third- or further lines of treatment for advanced lung cancer are usually based on each physician's experience. Thus, novel therapies are urgently required. The development of targeted therapies, including anlotinib, and immunotherapy has prompted a new era in the personalized management of lung cancer.

Han et al (37) performed a multicenter, randomized phase II clinical study (ALTER0302) of anlotinib and recruited 117 patients with refractory advanced NSCLC who failed at least 2 lines of chemotherapy or experienced drug intolerance. In the study, the 117 patients were randomized (1:1) to receive anlotinib (12 mg per day, per os; days 1-14; 21 days per cycle) or placebo. Anlotinib treatment significantly improved progression-free survival (PFS) compared with placebo [4.8 months vs. 1.2 months; hazard ratio $(\mathrm{HR})=0.32 ; 95 \% \mathrm{CI}, 0.20-0.51 ; \mathrm{P}<0.0001]$. The overall response rate (ORR) of the anlotinib group was better than that of the placebo group $(10.0 \% ; 95 \% \mathrm{CI}, 2.40-17.60 \%$ vs. $0 \%$; $95 \% \mathrm{CI}, 0-6.27 \%$; $\mathrm{P}=0.0280)$. The median overall survival (OS) time was 9.3 and 6.3 months for the anlotinib and placebo groups, respectively $(\mathrm{HR}=0.78 ; 95 \% \mathrm{CI}$, $0.51-1.18 ; \mathrm{P}=0.2316)$. The small sample size may be responsible for the failure to achieve statistical significance for the median OS. AEs were markedly more frequent in the anlotinib group than in the placebo group; the percentage of treatment-associated grade 3 or 4 AEs was $21.67 \%$. The most common treatment-associated grade 3 or 4 AEs were hypertension, elevated thyroglobulin (TG) and HFSR.

Regarding the efficacy of anlotinib in SCLC, another multicenter, randomized, double-blind phase II trial was performed. Cheng et al (38) investigated anlotinib vs. placebo for the third- or further-line treatment of SCLC (ALTER1202, NCT03059797). In the trial, 120 enrolled patients with limited- or extensive-stage SCLC who failed at least 2 lines of chemotherapy were randomized at a 2:1 ratio to the anlotinib arm or placebo arm (12 mg per os QD from day 1 to 14, every 3 weeks). PFS was the primary endpoint and the secondary endpoints included OS, the ORR, the disease control rate (DCR), quality of life (QoL) and safety. The outcomes demonstrated that the median PFS of the anlotinib arm was significantly prolonged compared with that of the placebo arm (4.1 months vs. 0.7 months; HR=0.19; 95\% CI, 0.12-0.32; $\mathrm{P}<0.0001)$. The ORR data were similar between the two groups; however, the DCR was higher in the anlotinib arm than in the placebo arm $(71.6 \%$ vs. $13.2 \%, \mathrm{P}<0.0001)$.

In summary, the above studies indicated that anlotinib has substantial clinical effects in patients with advanced NSCLC and SCLC. 


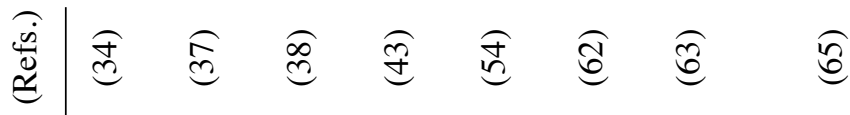

它莞

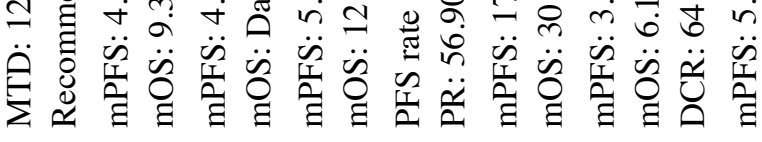

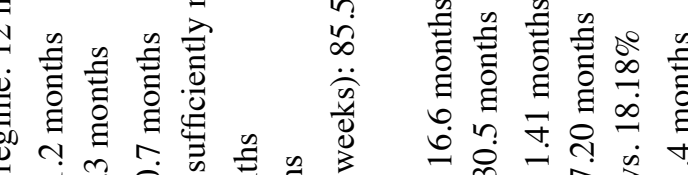

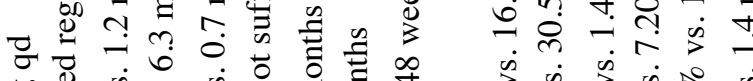

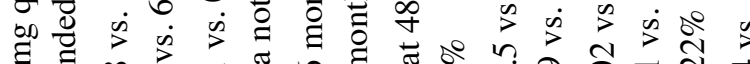

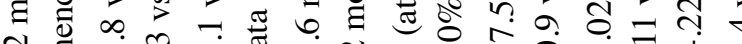

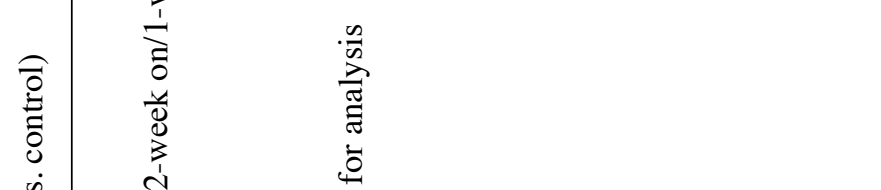

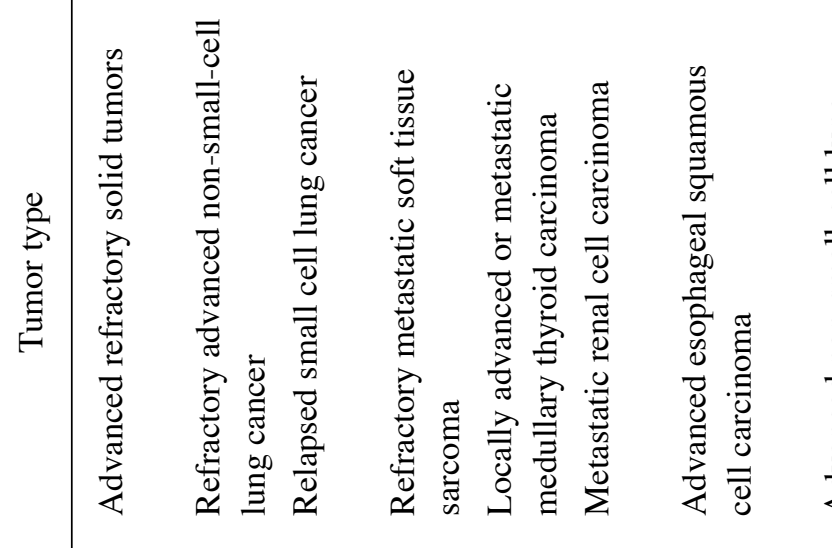

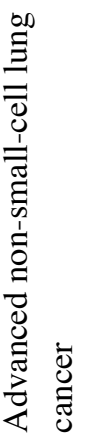

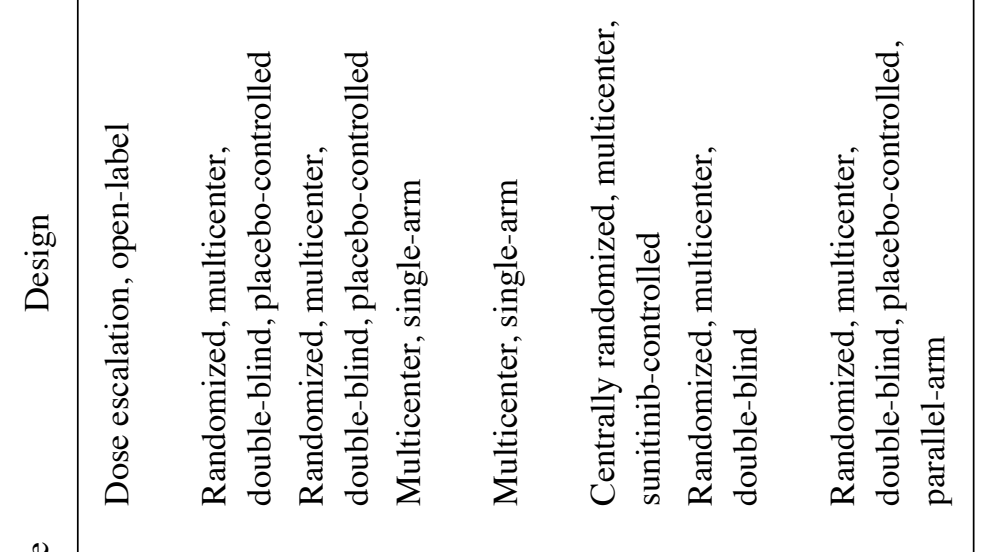

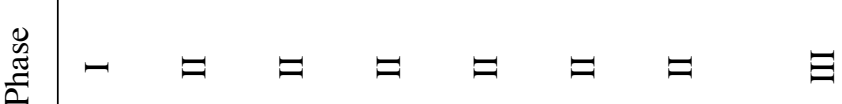


Anlotinib for advanced STS. STS comprises a group of relatively uncommon tumors, accounting for $\sim 1 \%$ of all solid malignancies $(39,40)$. Novel therapeutic options, including pazopanib, eribulin and trabectedin, have been developed in recent years. Unfortunately, none of them have been approved for STS treatment in China. In addition, the rarity of this disease and its numerous subtypes make obtaining large-scale data to guide treatment difficult. The prognosis of this disease remains poor, with a median OS of just over a year $(41,42)$. A phase I study of anlotinib conducted by Sun et al (34) demonstrated promising anti-tumor potential against STS. Based on the results, a multicenter, phase II study by Chi et al (43) (NCT01878448) investigated single-agent anlotinib in patients with STS who had disease progression after anthracycline-based first-line chemotherapy. A total of 166 patients were included in the final analysis and the participants were treated with oral anlotinib $12 \mathrm{mg}$ once daily, 2 weeks on/1 week off. The primary endpoint, the progression-free rate at 12 weeks $\left(\mathrm{PFR}_{12 \text { weeks }}\right)$, was $68 \%$, while the ORR was $13 \%$ (95\% CI, 7.6-18.0\%). The median PFS and OS were 5.6 and 12.0 months, respectively. Several histologic types of STS, such as fibrosarcoma, ASPS, liposarcoma and synovial sarcoma, were highly sensitive to anlotinib, with a $\mathrm{PFR}_{12 \text { weeks }}$ exceeding $70 \%$. The $\mathrm{PFR}_{12 \text { weeks }}$ and median PFS and OS for liposarcoma were 63\%, 5.6 months and 13.0 months, respectively, demonstrating promising clinical application value. Of note, anlotinib prolonged the median PFS of ASPS to 21 months, revealing a marked benefit of anlotinib.

The same research group further explored the association between age, prior lines of therapy and dose modification and the efficacy of anlotinib in advanced STS by conducting a randomized phase IIB trial (ALTER0203, NCT02449343) (44). A total of 158 patients were enrolled in the study. The results demonstrated a similar median PFS in patients receiving anlotinib who had received 0 or 1 prior lines of therapy and those who had received $2+$ prior lines of therapy (6.70 months vs. 6.33 months). The median PFS in patients aged $<65$ years was similar to that in patients aged $\geq 65$ years ( 6.33 and 5.90 months, respectively). Importantly, the median PFS was markedly prolonged in patients requiring $\geq 1$ dose reduction compared with those with no reduction (10.43 and 5.73 months, respectively). This trial further confirmed the single-agent activity of anlotinib in advanced STS.

Due to the potent therapeutic effect of anlotinib, it was recommended in the Chinese Society of Clinical Oncology guidelines for the treatment of STS in 2019 (45). Anlotinib received its second approval as a second-line treatment for advanced ASPS, clear cell sarcoma and other types of advanced STS after anthracycline-based first-line chemotherapy in June 2019 in the People's Republic of China (46).

Anlotinib for advanced MTC. Thyroid cancer is the ninth most common type of cancer worldwide, with an estimated 567,000 cases in 2018 (1). MTC is a rare form of cancer originating from thyroid parafollicular $\mathrm{C}$ cells and accounts for $2-3 \%$ of cases of thyroid gland neoplasia (47). Total thyroidectomy is the only curative treatment for MTC. Unfortunately, treatment options for distant metastatic MTC are limited, and conventional chemotherapy has unsatisfactory efficacy and considerable toxicity. The 10-year OS rate of metastatic MTC is $\sim 40 \%$ (48). Mutations in the Ret proto-oncogene are closely linked to the development of MTC (49). Systemic therapies, including multitargeted kinase inhibitors, are promising for advanced MTC. Several tyrosine kinase inhibitors (TKIs), such as vandetanib, cabozantinib, motesanib and sorafenib, have demonstrated promising clinical efficacy in unresectable MTC (50-53).

A single-arm, multicenter phase II clinical trial was performed in patients with unresectable or metastatic MTC (NCT01874873) (54). A total of 58 patients were enrolled and treated with $12 \mathrm{mg}$ anlotinib once daily, 2 weeks on/1 week off. The reported ORR and DCR were 56.90 and $93.10 \%$, respectively. The survival rates for the participants at 12,24 and 36 months were 89.7, 78.6 and 76.4\%, respectively. In addition, the PFS rates at 24, 36 and 48 weeks were 92.2, 87.8 and $84.5 \%$, respectively. Collectively, the results suggested that anlotinib exerts promising efficacy against advanced MTC.

Anlotinib for metastatic RCC ( $m R C C$ ). RCC is responsible for $>90 \%$ of neoplasms arising from the kidney. The clinical features of RCC are obscure and not specific in most cases. The only way to cure RCC is surgical resection. The five-year survival rate in patients with localized RCC is $292.5 \%$; however, it decreases to $69.6 \%$ in patients with regional disease and further decreases to $12.0 \%$ in patients with mRCC (55). $\mathrm{mRCC}$ is not sensitive to chemotherapy or radiotherapy. The past decade has witnessed marked advances in the treatment of mRCC, including targeted therapies with sunitinib, pazopanib, temsirolimus, sorafenib and bevacizumab plus interferon- $\alpha$ as the first- or second-line treatment (56-60). The sequential application of targeted drugs has proven to be a successful standard procedure for patients with $\mathrm{mRCC}$ with a failed previous therapy.

A multicenter phase II clinical trial conducted by Zhou et al (61) (NCT02072044) assessed the efficacy of anlotinib in $\mathrm{mRCC}$ patients who progressed or were intolerant to sorafenib or sunitinib. In the study, of the 43 enrolled patients, 42 were eligible and received treatment. One patient withdrew prior to treatment, 30 patients had disease progression and 10 were intolerant to sorafenib or sunitinib therapy. The median PFS for the whole group and for patients who had progressed on previous TKI treatment was 11.8 and 8.5 months, respectively. For intent-to-treat patients, the ORR was $19.10 \%$ (95\% CI, 8.60-34.12) and the 6-week DCR was $90.50 \%$ (95\% CI, 77.40-97.30\%). The results of this clinical trial demonstrating the excellent therapeutic effect of anlotinib were presented at an annual meeting of the American Society of Clinical Oncology in 2016 (61).

The same study group further performed a multicenter randomized phase II trial (NCT02072031) to compare the efficacy of anlotinib vs. sunitinib as the first-line treatment for patients with mRCC (62). A total of 133 patients (90 treated with anlotinib, 43 treated with sunitinib) were enrolled. Patients in the anlotinib group received an oral dose of $12 \mathrm{mg}$ once daily (2 weeks on/1 week off) and patients in the sunitinib group received an oral dose of $50 \mathrm{mg}$ once daily (4 weeks on/2 weeks off). The results demonstrated that both groups had similar PFS rates (17.5 months vs. 16.6 months, $\mathrm{P}>0.05$ ), median OS rates (30.9 months vs. 30.5 months, $\mathrm{P}>0.05$ ), ORRs (30.3\% vs. $27.9 \%$ ) and 6-week DCRs (97.8\% vs. $93.0 \%$, 
$\mathrm{P}=0.33$ ). Anlotinib is a potential first- or second-line treatment for patients with mRCC and has good prospects in clinical applications.

Anlotinib for advanced ESCC. Esophageal cancer ranks seventh in terms of incidence and sixth in terms of mortality, with 572,034 new cases of esophageal cancer and 508,585 deaths estimated in 2018, more than half of which occurred in China (1). The two major histological subtypes of esophageal cancer are esophageal adenocarcinoma and ESCC. ESCC accounts for $\sim 95 \%$ of all cases of esophageal cancer. Radiotherapy has a crucial role in the treatment of ESCC and it may be combined with chemotherapy for inoperable patients. However, acquired chemo/radioresistance may be the major reason for local recurrence or treatment failure. Novel drugs are therefore urgently required.

A randomized, double-blind, multicenter phase II trial by Huang et al (63) investigated single-agent anlotinib in patients with stage IV ESCC who had previously received at least one line of platinum- or taxane-based chemotherapy and experienced disease progression after chemotherapy (ALTER1102, NCT02649361). Patients were randomized (2:1) to receive anlotinib or placebo; 109 patients received anlotinib, while 55 patients received the placebo. The results suggested that patients treated with anlotinib had a significantly longer median PFS (3.02 months vs. 1.41 months; HR=0.46, 95\% CI: $0.32-0.66 ; \mathrm{P}<0.0001)$ than those treated with the placebo. The anlotinib group exhibited a markedly improved DCR compared with the placebo group $(62.44 \%$ vs. $18.18 \%$; $\mathrm{P}<0.0001)$. The above results were presented at the 2019 Gastrointestinal Cancers Symposium which was organized by the American Gastroenterological Association and American Society of Clinical Oncology (63).

Due to the promising therapeutic effect of anlotinib, it was recommended in the Chinese Society of Clinical Oncology guidelines for the treatment of advanced ESCC in 2019 (64).

Phase III. As discussed above, anlotinib has exhibited encouraging clinical activity, bringing new hope to patients with advanced NSCLC. A multicenter, double-blind, randomized phase III clinical trial (ALTER0303, NCT02388919) was performed to estimate the drug's efficacy in patients with advanced NSCLC who failed at least 2 lines of prior therapies (65). Patients harboring EGFR mutations or anaplastic lymphoma kinase (ALK) rearrangements must have failed at least 1 line of chemotherapy and TKI therapy to be enrolled in the study. A total of 437 patients from 31 grade-A tertiary hospitals were randomized (2:1) to receive oral anlotinib $12 \mathrm{mg}$ or matched placebo on a 2-week on/1-week off regime until progression or intolerable toxicity. Regarding efficacy, patients treated with anlotinib had significantly longer OS and PFS than those treated with placebo [OS: 9.6 months vs. 6.3 months, HR=0.68 (95\% CI, 0.54-0.87), $\mathrm{P}=0.002$; PFS: 5.4 months vs. 1.4 months, HR=0.25 (95\% CI, 0.19-0.31), $\mathrm{P}<0.001]$. The ORR and DCR were also substantially higher in the anlotinib group than in the placebo group [ORR, 27 (9.2\%) vs. 1 (0.7\%), P<0.001; DCR, 238 (81.0\%) vs. 53 (37.1\%), $\mathrm{P}<0.001]$.

The same study group further performed an exploratory subgroup analysis of the ALTER0303 trial to investigate the efficacy of anlotinib as a third-line treatment in patients with different EGFR gene statuses. The results revealed that anlotinib significantly improved PFS and OS in EGFR-mutated patients [OS: HR=0.59 (95\% CI, 0.37-0.93); PFS, HR=0.15 (95\% CI, 0.09-0.24)] and wild-type EGFR patients [OS: HR=0.73 $(95 \%$ CI, 0.55-0.97); PFS: HR=0.29 (95\% CI, 0.22-0.39)]. Patients with NSCLC bearing EGFR mutations or ALK rearrangements exhibited obvious responses to several TKIs, including gefitinib, erlotinib and crizotinib, in the initial stage (66). Commercially available means of detecting EGFR mutations and ALK rearrangements will facilitate the prediction of the drug response and enable the selection of an individualized treatment regime. However, the efficacy of erlotinib and gefitinib for EGFR unselected or wild-type patients is unsatisfactory. The ALTER0303 trial indicated that in both the EGFR-mutant and wild-type groups, anlotinib resulted in improved PFS and OS compared with the placebo group. In addition, a study by the same group suggested that patients with sensitive EGFR mutations had a significantly prolonged OS time compared with patients with wild-type EGFR when treated with anlotinib $[10.70$ vs. 8.87 months, $\mathrm{HR}=0.685,95 \%$ CI $0.50-0.95$, $\mathrm{P}=0.0204]$ (67). A retrospective study by Shao et al (68) determined that the median PFS in the EGFR-mutated and wild-type groups treated with anlotinib was 3.5 and 2.8 months, respectively, and there was no significant difference between the two groups $(\mathrm{P}=0.873)$. The aforementioned study did not demonstrate significant differences in the median PFS between the EGFR-mutated and wild-type groups, which may be due to the small sample size or because it was a retrospective single-center study; furthermore, with the inclusion of patients with different histological subtypes, there a certain heterogeneity was present in the study. Although patients with NSCLC harboring EGFR mutations may achieve prolonged survival and a preserved QoL, resistance invariably develops. The T790M mutation is mainly responsible for acquired drug resistance $(66,69,70)$. In the ALTER0303 trial, 18 patients with T790M benefited from anlotinib compared with placebo, with a median OS of 21.5 and 6.6 months, respectively (71).

Furthermore, in patients with adenocarcinoma, anlotinib provided benefits for both OS and PFS [OS: HR=0.67 $(95 \%$ CI, 0.51-0.89); PFS: HR=0.21 (95\% CI, 0.15-0.28)]. In patients with squamous cell carcinoma, a substantial increase in only PFS was achieved [HR=0.37 (95\% CI, 0.22-0.60)]. Post-hoc analysis revealed that a larger proportion of patients in the placebo group received subsequent treatment [93 (65.0\%) vs. 143 (48.6\%); $\mathrm{P}=0.002$ ], particularly chemotherapy [59 (41.3\%) vs. $66(22.5 \%) ; \mathrm{P}<0.001]$, than those in the anlotinib group.

The researchers also assessed QoL in the participants based on the ALTER0303 trial $(65,72)$. QoL was assessed using the European Organization for Research and Treatment of Cancer (EORTC) Quality of Life Questionnaire Core 30 (QLQ-C30) and the associated EORTC Quality of Life Lung Cancer Specific Module (QLQ-LC13). The results suggested that anlotinib improved the role functioning, social functioning, dyspnea, insomnia, constipation and financial situation of patients with advanced NSCLC compared with placebo. However, the anlotinib group exhibited worse sore mouth or tongue symptoms than the placebo group. The above data indicated that anlotinib improves QoL in patients with advanced NSCLC. 
Recently, the effect of anlotinib on the brain metastasis (BM) of NSCLC was reported on the basis of the ALTER0303 trial (73). The results indicated that more cases of BM were present among young patients and those with adenocarcinoma. Anlotinib contributed significantly to PFS (BM: $H R=0.19$, 0.11-0.34; non-BM: $\mathrm{HR}=0.29,0.22-0.37$; interaction $\mathrm{P}=0.691$ ) and $\mathrm{OS}$ (BM: $\mathrm{HR}=0.71,0.44-1.16$; non-BM: $\mathrm{HR}=0.67$, 0.51-0.89: Interaction $\mathrm{P}=0.789$ ) in patients with NSCLC over placebo in the BM and non-BM groups. In addition, anlotinib markedly prolonged the time to brain progression $(\mathrm{HR}=0.11$, 95\% CI 0.03-0.41, $\mathrm{P}=0.001$ ), which demonstrated that the risk of BM was reduced by $90 \%$ with anlotinib.

Subsequently, the same research group preliminarily investigated the possible factors that affected PFS and OS after anlotinib treatment in patients with refractory NSCLC. The data were also collected from the ALTER0303 trial (74). Multivariate analysis revealed that a high posttherapeutic peripheral blood granulocyte/lymphocyte ratio and elevated alkaline phosphatase levels were independent risk factors for PFS. Elevated thyroid-stimulating hormone (TSH), blood glucose and triglyceride levels, hypertension and HFSR were considered independent favorable predictors of PFS. Furthermore, a high posttherapeutic peripheral blood granulocyte/lymphocyte ratio, an Eastern Cooperative Oncology Group score of $\geq 2$ and the sum of the maximal target lesion length at baseline were independent risk factors for OS, whereas hypertriglyceridemia was an independent favorable predictor of OS.

Based on the results of the ALTER0303 trial, anlotinib was approved as a third-line treatment for patients with refractory advanced NSCLC after $\geq 2$ lines of systemic chemotherapy in China.

Ongoing trials. There are numerous ongoing trials with anlotinib for molecular targeted treatment in advanced NSCLC, STS, ESCC, gastric cancer, CRC, head and neck carcinoma, breast cancer and ovarian cancer. Selected ongoing trials with anlotinib are summarized in Table II. For example, a randomized, exploratory, open clinical Phase II trial has been designed to compare the efficacy and safety of anlotinib plus irinotecan vs. irinotecan in patients with ESCC (NCT03387904). Phase II and phase III trials investigating anlotinib in second- or further-line treatment for ovarian and gastric cancer are also actively recruiting (NCT03924882; NCT02461407).

\section{Summary of AEs associated with anlotinib in the treatment of advanced cancers}

The toxicities of anlotinib were acceptable or manageable in clinical trials and real-world clinical cases of patients with advanced cancers. During the phase I clinical trial of 21 patients treated with anlotinib (12 mg/day, 2/1 schedule) (34), hypothyroidism, triglyceride elevation, total cholesterol elevation, alanine aminotransferase elevation, diarrhea and proteinuria were the most commonly observed nonhematologic AEs. The hematologic toxicities that patients experienced were mild. The most frequently observed serious AEs reported in $>5 \%$ of patients were hypertension $(10 \%)$, triglyceride elevation $(10 \%)$, HFSR $(5 \%)$ and lipase elevation (5\%) (as presented in Fig. 2) (34).
Anlotinib also exhibited acceptable tolerability in a phase II clinical trial (37). Approximately $91.67 \%$ of patients in the anlotinib group experienced grade 1-4 AEs. The study indicated that the major AEs were hypertension $(55.0 \%)$, elevated TSH (36.7\%), HFSR (28.3\%), elevated TG (26.7\%), elevated total cholesterol $(25.0 \%)$ and diarrhea $(23.3 \%)$. The grade 3 or 4 AEs that occurred in $21.67 \%$ of patients included hypertension (10.0\%), elevated TG $(5.0 \%)$ and HFSR $(3.3 \%)$. In the anlotinib group, 6 out of 60 patients (10\%) had a dose reduction to $10 \mathrm{mg}$ per day due to toxicity. The major reasons for dose reduction were hypertension, HFSR, elevated liver enzymes and stomatitis.

The data from a phase III trial further confirmed the tolerability of anlotinib in advanced NSCLC (65). Hypertension, fatigue, TSH elevation, anorexia, hypertriglyceridemia, HFSR and hypercholesterolemia were the most common AEs in the anlotinib group. Approximately $61.9 \%$ of patients experienced grade 3 or higher AEs, which included hypertension (13.6\%), hyponatremia $(8.2 \%)$ and elevated $\gamma$-glutamyltransferase (5.4\%). The rates of grade 3 or higher AEs were 61.9 and $37.1 \%$ in the anlotinib and placebo arm, respectively. Approximately $8.2 \%$ of the patients treated with anlotinib had a dose reduction from 12 to $10 \mathrm{mg} / \mathrm{day}$, and $0.7 \%$ of the patients in the anlotinib group had a dose reduction from 12 to $8 \mathrm{mg} /$ day. The dose adjustment was mainly because of HFSR and hypertension.

\section{Management of drug-associated AEs}

General. Anlotinib has been proven to have promising efficacy in a range of advanced cancer types. However, it is also associated with various AEs that may affect the QoL of patients and hinder patient compliance to treatment. Studies have indicated that the percentage of AEs (all grades) that occurred in anlotinib-treated patients was $87.70-100 \%$, and grade 3 or higher AEs were reported in 21.67-61.90\% of anlotinib-treated patients $(34,37,38,62,65)$, and the percentages differed among the clinical trials. The most common AEs associated with anlotinib treatment included hypertension, HFSR, fatigue, diarrhea and anorexia. Therefore, the prevention and management of AEs during treatment with anlotinib are important for optimizing therapeutic benefits and avoiding unnecessary dose modifications or discontinuations.

Hypertension. Hypertension is generally acknowledged as a common AE of angiogenesis inhibitors, particularly VEGFR-TKIs (75). Clinical data have indicated that $42-55 \%$ of patients receiving anlotinib for advanced cancers developed hypertension (any grade) and 4.80-13.60\% of patients experienced grade 3 or higher hypertension $(37,43,54,62,65)$.

First, blood pressure (BP) assessment is required for patients who are normotensive or hypertensive, and BP should be controlled before treatment is initiated $(76,77)$. Frequent monitoring of BP during the first few months (weekly during the first cycle and subsequently every 2 to 3 weeks) is important for early intervention and the resolution of hypertension. The BP of patients who receive anti-hypertensive treatment should be measured twice daily. Monitoring BP may be accomplished at visits to the clinic or by self-monitoring at home.

Furthermore, patient education should be emphasized (78). Patients should learn which foods to eat and which to avoid. 


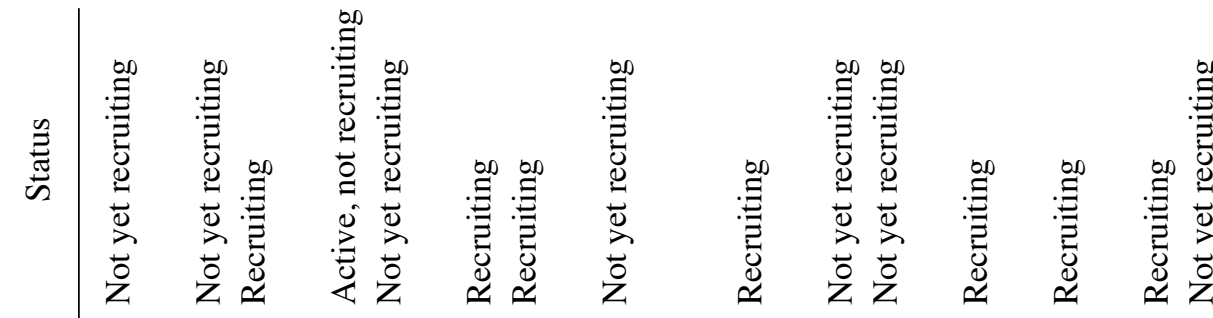

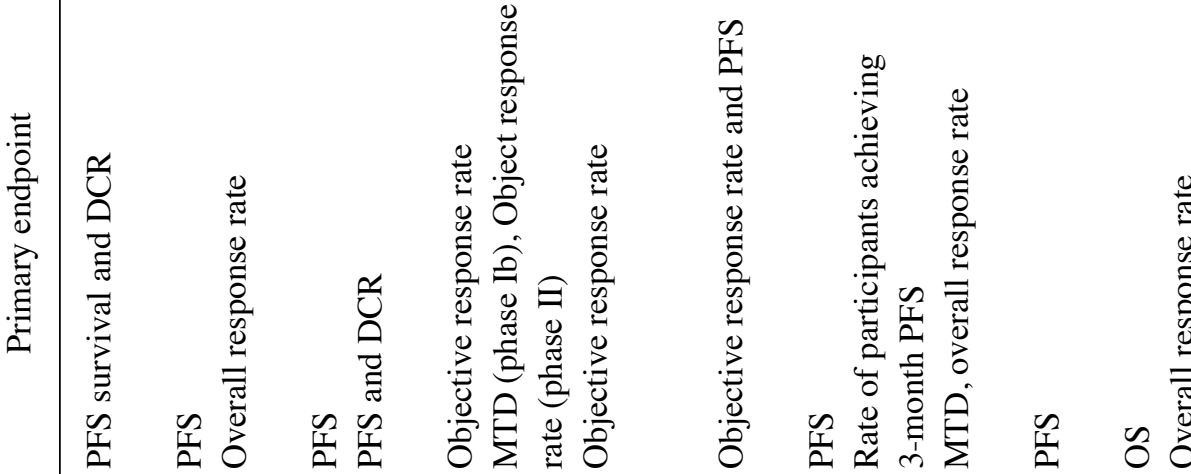

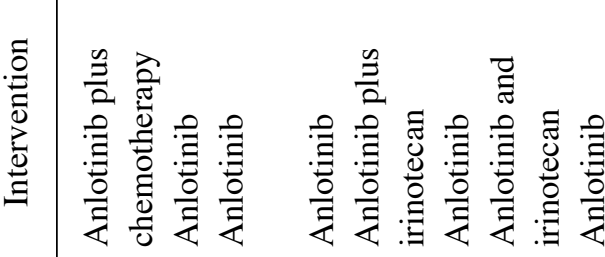

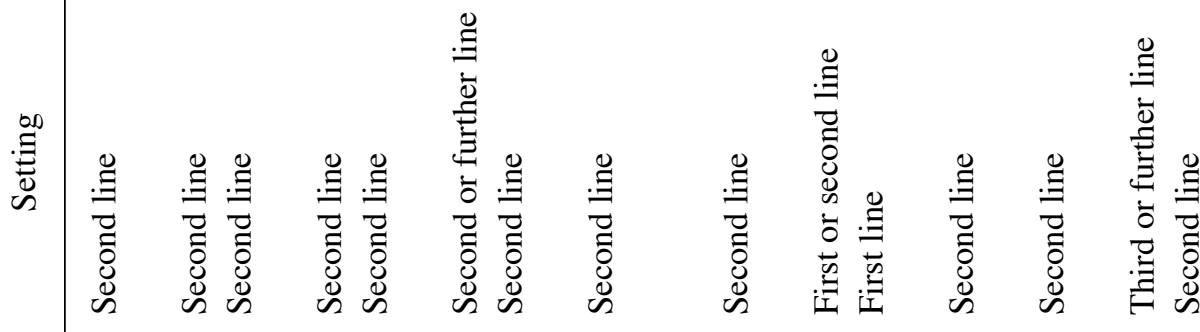

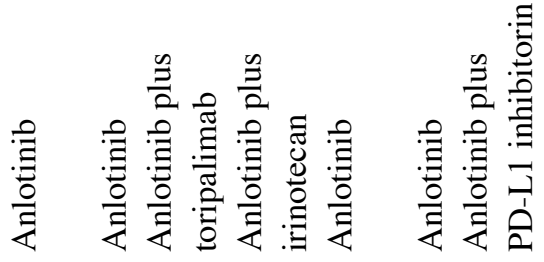

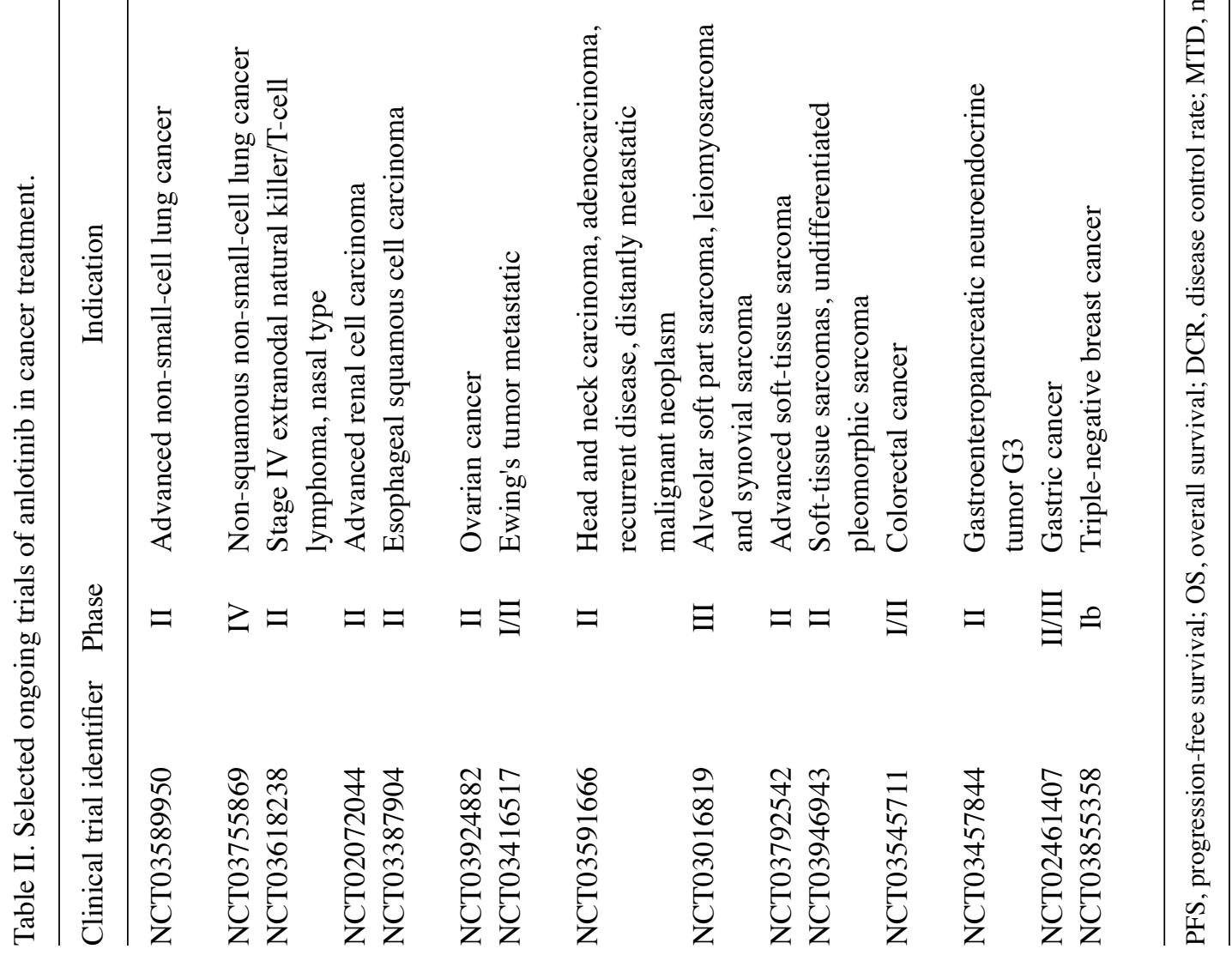




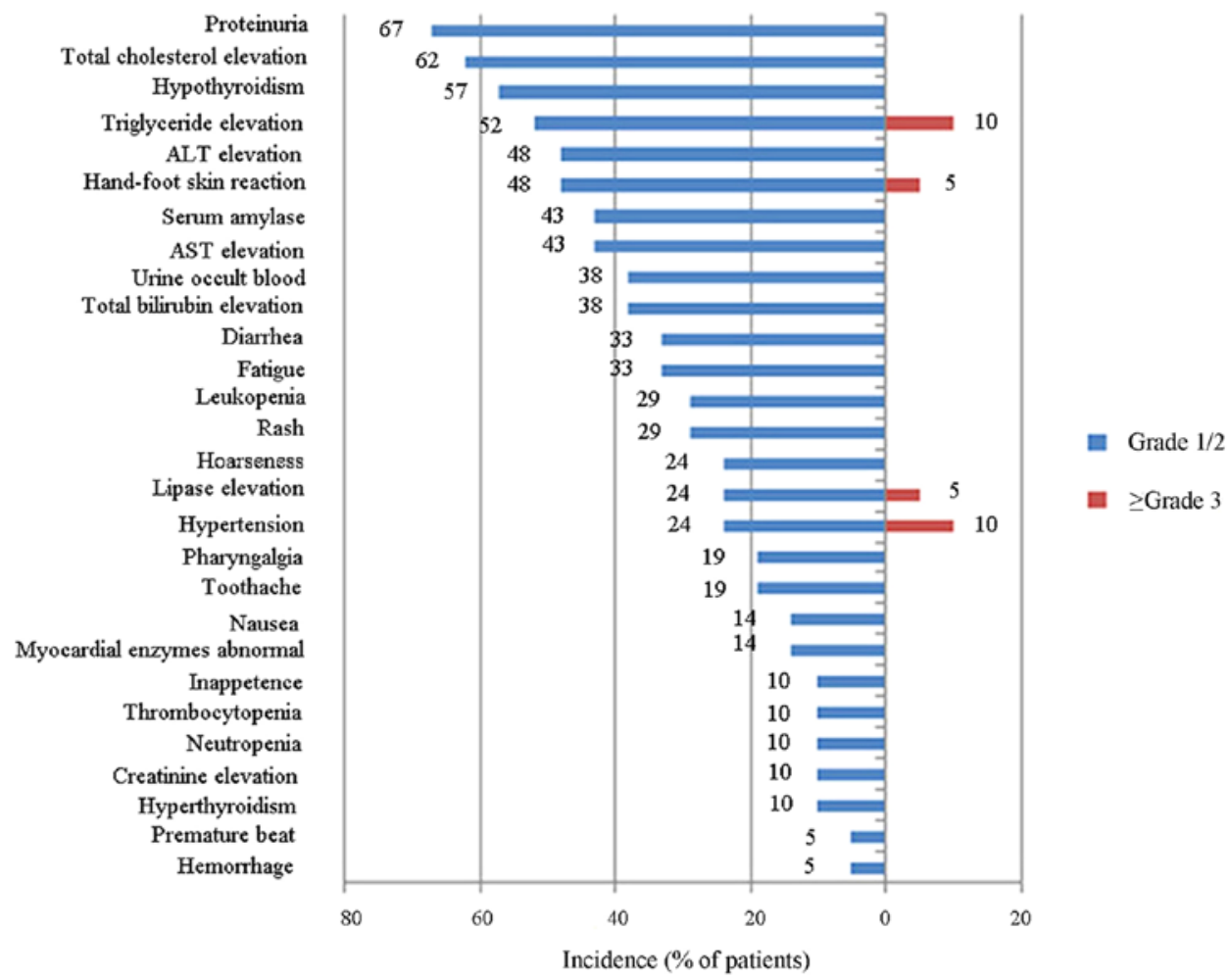

Figure 2. Adverse events in patients who received $12 \mathrm{mg} /$ day anlotinib on the 2-week-on/1-week-off schedule. ALT, alanine aminotransferase; AST, aspartate transaminase.

Patients should also be encouraged to report any symptoms of headaches, shortness of breath or blushing to their health care team.

In addition, anti-hypertensive drugs $(76,78)$, such as thiazide-type diuretics, angiotensin-converting enzyme inhibitors (ACEIs), angiotensin receptor blockers (ARBs), beta blockers and calcium channel blockers (CCBs), are all suitable choices for patients who develop hypertension during treatment. These drugs may be used alone or in combination, but the combination of ACEIs and ARBs is not recommended. Anlotinib is metabolized by cytochrome P450 family 3 subfamily A member 4 (CYP3A4). Diltiazem and verapamil are liver enzyme CYP3A4 inhibitors that should be avoided to be used together with anlotinib owing to potential drug interactions. In addition, nifedipine was reported to increase VEGF secretion; thus, the use of the dihydropyridine CCB nifedipine should also be avoided (79). Thiazide diuretics should be used carefully due to the risk of diarrhea. The choice of pharmacologic therapy is mainly dependent on the patient's medical history and clinical status. The BP may normalize upon the withdrawal of therapy and anti-hypertensive medication will accordingly need to be adjusted or discontinued.

HFSR. HFSR, also known as palmar-plantar erythrodysesthesia or hand-foot syndrome, is a common AE caused by certain chemotherapies or targeted therapies that may lead to physical and psychosocial discomfort (80). HFSR of any grade occurred in $28.33-79.31 \%$ of patients treated with anlotinib and grade 3 or higher was reported in 3.30-8.62\% of patients $(34,37,43,54,62)$. HFSR manifests with erythema, dysesthesia, pain, cracking and desquamation in pressure and flexure areas, such as the palms of hands or soles of feet (81). In most cases, HFSR is reversible. However, it is associated with a decrease in QoL and leads to undesirable results during treatment. Various measures may be taken to prevent and reduce the risk of anlotinib-induced HFSR $(76-78,81,82)$.

First, patient education, early recognition and regular follow-up in the first few months have vital roles in the management of HFSR. Furthermore, preventive measures should be taken prior to treatment. Patients should be advised to avoid mechanical stress and to wear thick cotton socks, gloves and comfortable shoes to protect their hands and feet. Skin care should be emphasized and urea-containing emollients are recommended. Cold or hot stimuli should be prevented and patients should ensure that their hands are dried after washing.

Furthermore, once HFSR develops, the above preventive measures should be continued. For patients with grade 1 HFSR, emollients containing 20-40\% urea should be used and regular follow-up (every two weeks) should be performed. For patients with grade 2 or 3 HFSR, topical treatments (cortisone or $0.05 \%$ clobetasol), oral analgesia and antibiotics may relieve or cure this AE. If the above methods are ineffective, dose modification and/or discontinuation may be considered to bring HFSR to grade $\leq 1$. If the severity decreases to grade $\leq 1$, the full dose may be resumed. If this results in the worsening of HFSR, dose reduction or interruption may be adopted in the subsequent treatment.

Fatigue. Fatigue of any grade was reported in 31.67-41.38\% of patients receiving anlotinib and grade 3 or higher fatigue occurred in $0-1.67 \%$ of patients $(34,37,43,54)$. 
Patient education on early recognition and reporting is essential for fatigue management. Fatigue may result from drug-associated side effects or cancer progression. Other potential contributors to fatigue, such as emotional distress, depression, anxiety, poor sleep patterns, pain, anemia and endocrine disorders, should be evaluated and treated as required (76-78,82). In addition, control of other AEs, including diarrhea, vomiting, nausea and weight loss, may also reduce fatigue. Patients are recommended to maintain proper hydration, have nutritious diets and incorporate a resting period into their daily schedule. Evidence suggests that home-based exercise is able to improve fatigue in patients with advanced lung cancer and CRC (83). Dose reduction should be performed only for patients with grade 3 or higher fatigue. Once fatigue is resolved, the full dose should be resumed immediately.

Diarrhea. Diarrhea is one of the most common side effects reported in TKI treatment and may lead to dose reduction or discontinuation during treatment. TKIs with broader target profiles have been reported to possibly be associated with a higher rate of diarrhea than agents that are highly specific for certain targets (84). The underlying mechanism of TKI-associated diarrhea has remained to be fully elucidated. Intestinal inflammation, mucosal injury and ion transport disorder may have roles in the development of diarrhea $(85,86)$. Diarrhea of any grade occurred in $23.33-34.48 \%$ of patients treated with anlotinib and grade 3 or higher diarrhea occurred in $1.10 \%$ of patients $(34,37,54,62)$.

Early recognition and reporting may help avoid severe diarrhea. When diarrhea develops, dietary adjustment is required $(77,82)$. Patients are encouraged to avoid certain foods, including caffeine, alcohol, lactose-containing products, as well as insoluble and high-fiber foods. Fluid intake should be increased to avoid dehydration. If diarrhea persists after dietary management, patients are advised to receive pharmacological intervention. Anti-diarrheal agents, such as loperamide and diphenoxylate/atropine, may be taken into consideration $(77,84)$. For patients who are unresponsive to medication therapy or for those with severe diarrhea, anlotinib dose modification or interruption may be considered; once diarrhea is resolved, the full dose should be resumed immediately.

Anorexia. Anorexia of any grade was experienced by $13.33-34.48 \%$ of patients treated with anlotinib, while grade 3 or higher anorexia seldomly occurred $(37,54,62)$. In numerous patients, the development of anorexia may be drug-related or due to extreme anxiety or depression. For dietary management, a nutritious and high-calorie diet is recommended, such as protein drinks, eggs, meat and dairy products. Appetite stimulants, such as dronabinol or megestrol acetate, may also be employed (77). In addition, instead of attempting to consume three large meals, patients are encouraged to have multiple small meals. Gentle exercise, such as walking, swimming or riding a bicycle, may also boost the appetite. Furthermore, the management of other treatment-related AEs, such as nausea and vomiting, may reduce anorexia. Patients with cancer are prone to developing anxiety or depression, so appropriate social activities are required. A dose reduction should only be considered in severe cases.

\section{Future directions}

Anlotinib has a dual role, exhibiting both anti-tumor and anti-angiogenic activities. Furthermore, it is a promising therapeutic option for additional types of solid cancer. However, there are still several problems to be addressed.

First, the identification of predictive biomarkers is particularly important for the selection of optimal candidates for targeted therapy, which also applies to anlotinib. Lu et al (87) found that the serum levels of kallikrein related peptidase 5 and L1 cell adhesion molecule were negatively correlated with the response of NSCLC to anlotinib administered as a third-line treatment. A study on biomarkers for anlotinib in NSCLC indicated that CD31-labeled activated circulating endothelial cells (aCECs) were a sensitive marker for predicting the treatment efficacy of anlotinib. Patients who received anlotinib treatment were stratified according to the ratio of minimum aCECs counts at each time points to baseline (aCECs min/baseline) as $<1$ or $>1$. The results revealed that patients with CD31-labeled aCECs min/baseline $<1$ had prolonged PFS (88). The same study group established a prediction model of the tumor mutation index (TMI) by integrating three predictors, namely the germline and somatic mutation burden, the nonsynonymous and synonymous mutation burden and an unfavorable mutation score of cell-free DNA and circulating tumor DNA profiles. The researchers demonstrated that patients with low TMI scores who lacked the isocitrate dehydrogenase [NADP $(+)]$ $1^{\text {exon4 }}$ mutation had relatively good treatment responses $(89,90)$. Chen et al (91) performed a retrospective study that revealed that tumor cavitation was an independent factor associated with improved PFS. Anlotinib caused hand-foot syndrome, hypertension and prolonged QT intervals, all of which are considered good predictors of PFS (74). However, most of the current potential biomarkers were identified from the ALTER0303 study, which had a limited study population. There is no definitive biomarker for monitoring the treatment response of anlotinib in patients with cancer as of yet. Thus, additional studies are still needed to identify validated biomarkers to predict individualized therapeutic responses.

Furthermore, current clinical studies of anlotinib in MTC, RCC, ESCC, gastric cancer and ovarian cancer have been completed or are ongoing. The tumor site and histology may influence the efficacy of anlotinib and further studies are required to confirm the optimal regimen for different cancer types $(37,43,65,74)$.

In addition, considering the limited benefit that patients may gain from anlotinib monotherapy treatments, various clinical trials are evaluating the combination of anlotinib and chemotherapy. For instance, several ongoing studies are investigating i) the efficacy and safety of anlotinib combined with docetaxel/pemetrexed and S-1 as a second-line treatment for subjects with advanced NSCLC (NCT03589950); ii) the efficacy and safety of anlotinib plus docetaxel as a second-line treatment for patients with wild-type advanced non-squamous NSCLC (NCT03750916); and iii) the efficacy and safety of anlotinib plus irinotecan as a second-line treatment for advanced ESCC (NCT03387904). Our research group plans to evaluate the efficacy and safety of anlotinib plus concurrent chemoradiotherapy as a second-line treatment for advanced ESCC. Certain studies have focused on the combination of immunotherapy and anti-angiogenic agents for 
the treatment of advanced NSCLC. Increasing evidence suggests that angiogenesis may be associated with immunosuppression in the tumor microenvironment, thereby mediating tumor evasion of immune system surveillance (92). Immunotherapy may be anti-angiogenic and anti-angiogenic agents may also stimulate the immune system. The combination of these two therapies may enhance both of these anti-tumor effects (93). The combined regimen with immunotherapy and anti-angiogenic agents, such as nivolumab plus bevacizumab, demonstrated tolerable treatment-associated toxicity and encouraging therapeutic effects in preliminary investigations (94). By contrast, the combination of several EGFR-TKIs, such as erlotinib, gefitinib and osimertinib, with immunotherapy was associated with relatively high incidence rates of toxicities (95). Therefore, this combined therapy is still under investigation. Thus far, there has been no report on the combination of anlotinib and immunotherapy. Finally, the feasibility of oral administration and relative safety of anlotinib indicate that it may serve as a maintenance therapy after first-line treatment, but this still requires to be explored in future clinical trials.

Drug resistance remains a major problem in the late stage of targeted therapy, which also applies to anlotinib. Despite the continuous efforts researchers have made in recent years to overcome this, few approaches have been discovered. The use of several miRs and supplementation with exogenous CXCL2 was reported to potentially circumvent anlotinib resistance $(31,33)$. Further efforts are anticipated to solve the drug resistance issue.

Finally, anlotinib resulted in prolonged PFS and OS in EGFR-mutant and wild-type patients, but there is a lack of data on the activity of anlotinib within two important clinically relevant molecular subgroups, the EGFR-mutant/ALK-positive and EGFR-wild-type/ALK-negative groups. In the future, more in-depth research is required in this area.

\section{Conclusion}

In the present study, preclinical and clinical trials of anlotinib were reviewed with a focus on safety evaluation and $\mathrm{AE}$ management. Anlotinib received its first approval as a third-line treatment for refractory advanced NSCLC in May 2018 and its second approval as a second-line treatment for advanced STS in June 2019 in China. As a second- or third-line therapy, anlotinib has demonstrated encouraging efficacy and a manageable and tolerable safety profile in a broad range of malignancies. The majority of anlotinib-associated AEs may be managed with patient education, prophylactic measures, dose modification and medical intervention. However, anlotinib should not be applied to patients with central squamous cell lung carcinoma, those at high risk of hemoptysis, those with severe hepatorenal insufficiency and those who are pregnant and lactating.

\section{Acknowledgements}

Not applicable.

\section{Funding}

This work was supported by the National Natural Science Foundation of China (grant nos. 31700737 and 31670857), the Innovative Research Program of Jiangsu Province (grant no. 2017ZXK7QW08) and the Young Talents Subsidy Project of Wuxi Municipal Health Commission (grant no. QNRC092).

\section{Availability of data and materials}

Not applicable.

\section{Authors' contributions}

YG contributed to the literature search and selected the studies for inclusion. YG and PL drafted the manuscript and revised it critically for important intellectual content; RS designed the study and revised the manuscript. All authors read and approved the final version of the manuscript.

\section{Ethics approval and consent to participate}

Not applicable.

\section{Patient consent for publication}

Not applicable.

\section{Competing interests}

The authors declare that they have no competing interests.

\section{References}

1. Bray F, Ferlay J, Soerjomataram I, Siegel RL, Torre LA and Jemal A: Global cancer statistics 2018: GLOBOCAN estimates of incidence and mortality worldwide for 36 cancers in 185 countries. CA Cancer J Clin 68: 394-424, 2018.

2. Viallard $C$ and Larrivée B: Tumor angiogenesis and vascular normalization: Alternative therapeutic targets. Angiogenesis 20: 409-426, 2017.

3. Voravud N and Charuruk N: Tumor angiogenesis. J Med Assoc Thai 82: 394-404, 1999.

4. Regad T: Targeting RTK signaling pathways in cancer. Cancers (Basel) 7: 1758-1784, 2015.

5. Zwick E, Bange J and Ullrich A: Receptor tyrosine kinase signalling as a target for cancer intervention strategies. Endocr Relat Cancer 8: 161-173, 2001

6. Petrelli F, Borgonovo K, Cabiddu M, Ghilardi M and Barni S: Cetuximab and panitumumab in KRAS wild-type colorectal cancer: A meta-analysis. Int J Colorectal Dis 26: 823-833, 2011.

7. Butti R, Das S, Gunasekaran VP, Yadav AS, Kumar D and Kundu GC: Receptor tyrosine kinases (RTKs) in breast cancer: Signaling, therapeutic implications and challenges. Mol Cancer 17: 34, 2018

8. Giaccone G, González-Larriba JL, van Oosterom AT, Alfonso R, Smit EF, Martens M, Peters GJ, van der Vijgh WJ, Smith R, Averbuch $\mathrm{S}$ and Fandi A: Combination therapy with gefitinib, an epidermal growth factor receptor tyrosine kinase inhibitor, gemcitabine and cisplatin in patients with advanced solid tumors. Ann Oncol 15: 831-838, 2004.

9. Manning G, Plowman GD, Hunter T and Sudarsanam S: Evolution of protein kinase signaling from yeast to man. Trends Biochem Sci 27: 514-520, 2002.

10. Knox JJ, Barrios CH, Kim TM, Cosgriff T, Srimuninnimit V, Pittman K, Sabbatini R, Rha SY, Flaig TW, Page RD, et al: Final overall survival analysis for the phase II RECORD-3 study of first-line everolimus followed by sunitinib versus first-line sunitinib followed by everolimus in metastatic RCC. Ann Oncol 28: 1339-1345, 2017. 
11. Ogasawara S, Chiba T, Ooka Y, Suzuki E, Maeda T, Yokoyama M, Wakamatsu T,Inoue M,Saito T,Kobayashi K, et al: Characteristics of patients with sorafenib-treated advanced hepatocellular carcinoma eligible for second-line treatment. Invest New Drugs 36 332-339, 2018.

12. How J, Mann J, Laczniak AN and Baggstrom MQ: Pulsatile erlotinib in EGFR-positive non-small-cell lung cancer patients with leptomeningeal and brain metastases: Review of the literature. Clin Lung Cancer 18: 354-363, 2017.

13. Zhou C, Wu YL, Chen G, Feng J, Liu XQ, Wang C, Zhang S, Wang J, Zhou S, Ren S, et al: Erlotinib vs. chemotherapy as first-line treatment for patients with advanced EGFR mutation-positive non-small-cell lung cancer (OPTIMAL, CTONG-0802): A multicentre, open-label, randomised, phase 3 study. Lancet Oncol 12: 735-742, 2011.

14. Urata Y, Katakami N, Morita S, Kaji R, Yoshioka H, Seto T, Satouchi M, Iwamoto Y, Kanehara M, Fujimoto D, et al: Randomized phase III study comparing gefitinib with erlotinib in patients with previously treated advanced lung adenocarcinoma: WJOG 5108L. J Clin Oncol 34: 3248-3257, 2016.

15. Liao Z, Li F, Zhang C, Zhu L, Shi Y, Zhao G, Bai X, Hassan S, Liu X, Li T, et al: Phase II trial of VEGFR2 inhibitor apatinib for metastatic sarcoma: Focus on efficacy and safety. Exp Mol Med 51: 1-11, 2019.

16. Sandler A, Gray R, Perry MC, Brahmer J, Schiller JH, Dowlati A, Lilenbaum R and Johnson DH: Paclitaxel-carboplatin alone or with bevacizumab for non-small-cell lung cancer. N Engl J Med 355: 2542-2550, 2006.

17. An $\mathrm{J}$ and Lv W: Endostar (rh-endostatin) versus placebo in combination with vinorelbine plus cisplatin chemotherapy regimen in treatment of advanced non-small cell lung cancer: A meta-analysis. Thorac Cancer 9: 606-612, 2018

18. Syed YY: Anlotinib: First global approval. Drugs 78: 1057-1062, 2018.

19. Liang L, Hui K, Hu C, Wen Y, Yang S, Zhu P, Wang L, Xia Y, Qiao Y, Sun W, et al: Autophagy inhibition potentiates the anti-angiogenic property of multikinase inhibitor anlotinib through JAK2/STAT3/VEGFA signaling in non-small cell lung cancer cells. J Exp Clin Cancer Res 38: 71, 2019.

20. Wang G, Sun M, Jiang Y, Zhang T, Sun W, Wang H, Yin F, Wang Z, Sang W, Xu J, et al: Anlotinib, a novel small molecular tyrosine kinase inhibitor, suppresses growth and metastasis via dual blockade of VEGFR2 and MET in osteosarcoma. Int J Cancer 145: 979-993, 2019.

21. Ruan X, Shi X, Dong Q, Yu Y, Hou X, Song X, Wei X, Chen L and Gao M: Antitumor effects of anlotinib in thyroid cancer. Endocr Relat Cancer 26: 153-164, 2019.

22. Lin B, Song X, Yang D, Bai D, Yao Y and Lu N: Anlotinib inhibits angiogenesis via suppressing the activation of VEGFR2, PDGFR $\beta$ and FGFR1. Gene 654: 77-86, 2018.

23. Xie C, Wan X, Quan H, Zheng M, Fu L, Li Y and Lou L: Preclinical characterization of anlotinib, a highly potent and selective vascular endothelial growth factor receptor-2 inhibitor. Cancer Sci 109: 1207-1219, 2018.

24. Jang HS, Woo SR, Song KH, Cho H, Chay DB, Hong SO, Lee HJ, Oh SJ, Chung JY, Kim JH and Kim TW: API5 induces cisplatin resistance through FGFR signaling in human cancer cells. Exp Mol Med 49: e374, 2017.

25. Turkington RC, Longley DB, Allen WL, Stevenson L, McLaughlin K, Dunne PD, Blayney JK, Salto-Tellez M, Van Schaeybroeck S and Johnston PG: Fibroblast growth factor receptor 4 (FGFR4): A targetable regulator of drug resistance in colorectal cancer. Cell Death Dis 5: e1046, 2014.

26. Sugimoto K, Miyata Y, Nakayama T, Saito S, Suzuki R, Hayakawa F, Nishiwaki S, Mizuno H, Takeshita K, Kato H, et al: Fibroblast Growth Factor-2 facilitates the growth and chemo-resistance of leukemia cells in the bone marrow by modulating osteoblast functions. Sci Rep 6: 30779, 2016.

27. Saito S, Morishima K, Ui T, Hoshino H, Matsubara D, Ishikawa S, Aburatani H, Fukayama M, Hosoya Y, Sata N, et al: The role of $\mathrm{HGF} / \mathrm{MET}$ and FGF/FGFR in fibroblast-derived growth stimulation and lapatinib-resistance of esophageal squamous cell carcinoma. BMC Cancer 15: 82, 2015.

28. Cochrane DR, Spoelstra NS, Howe EN, Nordeen SK and Richer JK: MicroRNA-200c mitigates invasiveness and restores sensitivity to microtubule-targeting chemotherapeutic agents. Mol Cancer Ther 8: 1055-1066, 2009

29. Bayraktar R and Van Roosbroeck K: miR-155 in cancer drug resistance and as target for miRNA-based therapeutics. Cancer Metastasis Rev 37: 33-44, 2018.
30. Wang Y, Zhao M, Zhao H, Cheng S, Bai R and Song M: MicroRNA-940 restricts the expression of metastasis-associated gene MACC1 and enhances the antitumor effect of Anlotinib on colorectal cancer. Onco Targets Ther 12: 2809-2822, 2019.

31. Ma DB, Qin MM, Shi L and Ding XM: MicroRNA-6077 enhances the sensitivity of patients-derived lung adenocarcinoma cells to anlotinib by repressing the activation of glucose transporter 1 pathway. Cell Signal 64: 109391, 2019.

32. Wang L, En H, Yang L, Zhang Y, Sun B and Gao J: miR-596 suppresses the expression of Survivin and enhances the sensitivity of osteosarcoma cells to the molecular targeting agent anlotinib. Onco Targets Ther 12: 6825-6838, 2019.

33. Lu J, Xu W, Qian J, Wang S, Zhang B, Zhang L, Qiao R, Hu M, Zhao Y, Zhao X, et al: Transcriptome profiling analysis reveals that CXCL2 is involved in anlotinib resistance in human lung cancer cells. BMC Med Genomics 12 (Suppl 2): S38, 2019.

34. Sun Y, Niu W, Du F, Du C, Li S, Wang J, Li L, Wang F, Hao Y, $\mathrm{Li} \mathrm{C}$ and Chi Y: Safety, pharmacokinetics, and antitumor properties of anlotinib, an oral multi-target tyrosine kinase inhibitor, in patients with advanced refractory solid tumors. J Hematol Oncol 9: 105, 2016.

35. Werner TL, Kannapel E, Chen J, Chen M and Cohen AL: Safety and PK results from a phase Ib study of AL3818 (anlotinib) hydrochloride in subjects with ovarian, cervical, and endometrial cancers. J Clin Oncol 35 (15 Suppl): e17071, 2017.

36. Reck M and Rabe KF: precision diagnosis and treatment for advanced non-small-cell lung cancer. N Engl J Med 377: 849-861, 2017.

37. Han B, Li K, Zhao Y, Li B, Cheng Y, Zhou J, Lu Y, Shi Y, Wang Z, Jiang L, et al: Anlotinib as a third-line therapy in patients with refractory advanced non-small-cell lung cancer: A multicentre, randomised phase II trial (ALTER0302). Br J Cancer 118: 654-661, 2018.

38. Cheng Y, Wang Q, Li K, Shi J, Wu L, Han B, Chen G, He J, Wang J, Qin H and Li X: Anlotinib as third-line or further-line treatment in relapsed SCLC: A multicentre, randomized, doubleblind phase 2 trial. J Thorac Oncol 13 (10 Suppl): S351-S352, 2018.

39. Morrison BA: Soft tissue sarcomas of the extremities. Proc (Bayl Univ Med Cent) 16: 285-290, 2003.

40. Gatta G, van der Zwan JM, Casali PG, Siesling S, Dei Tos AP, Kunkler I, Otter R, Licitra L, Mallone S, Tavilla A, et al: Rare cancers are not so rare: The rare cancer burden in Europe. Eur J Cancer 47: 2493-2511, 2011

41. Meyer M and Seetharam M: First-line therapy for metastatic soft tissue sarcoma. Curr Treat Options Oncol 20: 6, 2019.

42. Ryan CW, Merimsky O, Agulnik M, Blay JY, Schuetze SM, Van Tine BA, Jones RL, Elias AD, Choy E, Alcindor T, et al: PICASSO III: A phase III, placebo-controlled study of doxorubicin with or without palifosfamide in patients with metastatic soft tissue sarcoma. J Clin Oncol 34: 3898-3905, 2016.

43. Chi Y, Fang Z, Hong X, Yao Y, Sun P, Wang G, Du F, Sun Y, Wu Q, Qu G, et al: Safety and efficacy of anlotinib, a multikinase angiogenesis inhibitor, in patients with refractory metastatic soft-tissue sarcoma. Clin Cancer Res 24: 5233-5238, 2018.

44. Yao Y, Chi Y, Fang Z, Wang S, Huang G, Cai Q, Shang G, Wang G, Qu G, Wu Q, et al: Efficacy of anlotinib in advanced soft tissue sarcoma by prior lines of therapy, age and dose modification. Ann Oncol 29 (Suppl 8): viii576-viii595, 2018.

45. Niu X, Wang J XB, Yu S, Zhang X, Huang Z, Cai J, Cai Z, Chen J, Cheng X, et al: Chinese society of clinical oncology (CSCO) guidelines for diagnosis and treatment of soft tissue sarcoma. People's Medical Publishing House, Beijing, China, 1st edition, 2019.

46. Anlotinib Hydrochloride Capsules [Package insert]. Lianyungang, China: ChiaTai TianQing Pharmaceutical Group, 2019. (In Chinese)

47. Lim H, Devesa SS, Sosa JA, Check D and Kitahara CM: Trends in thyroid cancer incidence and mortality in the United States, 1974-2013. JAMA 317: 1338-1348, 2017.

48. Roman S, Lin R and Sosa JA: Prognosis of medullary thyroid carcinoma: Demographic, clinical, and pathologic predictors of survival in 1252 cases. Cancer 107: 2134-2142, 2006.

49. Romei C, Ciampi R and Elisei R: A comprehensive overview of the role of the RET proto-oncogene in thyroid carcinoma. Nat Rev Endocrinol 12: 192-202, 2016.

50. Wells SA Jr, Gosnell JE, Gagel RF, Moley J, Pfister D, Sosa JA, Skinner M, Krebs A, Vasselli J and Schlumberger M: Vandetanib for the treatment of patients with locally advanced or metastatic hereditary medullary thyroid cancer. J Clin Oncol 28: 767-772, 2010 . 
51. Kurzrock R, Sherman SI, Ball DW, Forastiere AA, Cohen RB, Mehra R, Pfister DG, Cohen EE, Janisch L, Nauling F, et al: Activity of XL184 (Cabozantinib), an oral tyrosine kinase inhibitor, in patients with medullary thyroid cancer. J Clin Oncol 29: 2660-2666, 2011.

52. Schlumberger MJ, Elisei R, Bastholt L, Wirth LJ, Martins RG, Locati LD, Jarzab B, Pacini F, Daumerie C, Droz JP, et al: Phase II study of safety and efficacy of motesanib in patients with progressive or symptomatic, advanced or metastatic medullary thyroid cancer. J Clin Oncol 27: 3794-3801, 2009.

53. Lam ET, Ringel MD, Kloos RT, Prior TW, Knopp MV, Liang J, Sammet S, Hall NC, Wakely PE Jr, Vasko VV, et al: Phase II clinical trial of sorafenib in metastatic medullary thyroid cancer. J Clin Oncol 28: 2323-2330, 2010.

54. Sun Y, Du F, Gao M, Ji Q, Li Z, Zhang Y, Guo Z, Wang J, Chen X, Wang J, et al: Anlotinib for the treatment of patients with locally advanced or metastatic medullary thyroid cancer. Thyroid 28 : $1455-1461,2018$

55. Howlader N, Noone AM, Krapcho M, Miller D, Brest A, Yu M, Ruhl J, Tatalovich Z, Mariotto A, Lewis DR, et al (eds): SEER Cancer statistics review, 1975-2016. National Cancer Institute, Bethesda, MD, 2018. https://seer.cancer.gov/csr/1975_2016/. Updated April 9, 2020.

56. Rini BI, Powles T, Atkins MB, Escudier B, McDermott DF, Suarez C, Bracarda S, Stadler WM, Donskov F, Lee JL, et al: Atezolizumab plus bevacizumab versus sunitinib in patients with previously untreated metastatic renal cell carcinoma (IMmotion151): A multicentre, open-label, phase 3, randomised controlled trial. Lancet 393: 2404-2415, 2019.

57. Motzer RJ, Hutson TE, Cella D, Reeves J, Hawkins R, Guo J, Nathan P, Staehler M, de Souza P, Merchan JR, et al: Pazopanib versus sunitinib in metastatic renal-cell carcinoma. N Engl J Med 369: 722-731, 2013.

58. Lee JB, Park HS, Park S, Lee HJ, Kwon KA, Choi YJ, Kim YJ, Nam CM, Cho NH, Kang B, et al: Temsirolimus in Asian metastatic/recurrent non-clear cell renal carcinoma. Cancer Res Treat 51: 1578-1588, 2019.

59. Liu C, Cao F, Xing W, Si T, Yu H, Yang X and Guo Z: Efficacy of cryoablation combined with sorafenib for the treatment of advanced renal cell carcinoma. Int J Hyperthermia 36: 220-228, 2019.

60. Schultze-Seemann W, Schulz H, Tschechne B and Häckl M: Bevacizumab plus IFN-alpha-2a in first-line treatment of patients with advanced or metastatic renal cell carcinoma: A prospective german non-interventional study. Anticancer Res 39: 875-882, 2019.

61. Zhou AP, Bai YX, Song V, Li HZ, Xie XD, Ren XB, Ye DW, Liu JY, Luo H, Bai XZ, et al: Anlotinib in metastatic renal cell carcinoma (mRCC) with a previous anti-VEGFR TKI: Preliminary results from a multicenter, phase II trial. J Clin Oncol 34: (15 Suppl): e16082, 2016

62. Zhou AP, Bai Y, Song Y, Luo H, Ren XB, Wang X, Shi B, Fu C Cheng Y, Liu J, et al: Anlotinib versus sunitinib as first-line treatment for metastatic renal cell carcinoma: A randomized phase II clinical trial. Oncologist 24: e701-e708, 2019.

63. Huang J, Xiao J, Fang W, Lu P, Fan Q, Shu Y, Feng JF, Zhang S, $\mathrm{Ba} \mathrm{Y}$, Liu Y, et al: Anlotinib in chemotherapy-refractory metastatic esophageal squamous cell carcinoma (ESCC): A randomized, double-blind, multicenter phase II trial. J Clin Oncol 37 (4 Suppl): S95, 2019.

64. Wang L HJ, Han Y, Li Y, Fu J, Mao W, Wang X, Chen K, Fang W, Fan Q, et al: Chinese Society of Clinical Oncology (CSCO) guidelines for diagnosis and treatment of esophageal cancer. People's Medical Publishing House, Beijing, China, 1st edition, 2019.

65. Han B, Li K, Wang Q, Zhang L, Shi J, Wang Z, Cheng Y, He J, Shi Y, Zhao Y, et al: Effect of anlotinib as a third-line or further treatment on overall survival of patients with advanced non-small cell lung cancer: The ALTER 0303 phase 3 randomized clinical trial. JAMA Oncol 4: 1569-1575, 2018.

66. Haber DA, Bell DW, Sordella R, Kwak EL, Godin-Heymann N, Sharma SV, Lynch TJ and Settleman J: Molecular targeted therapy of lung cancer: EGFR mutations and response to EGFR inhibitors. Cold Spring Harb Symp Quant Biol 70: 419-426, 2005.

67. Li K, Han BH, Wang QM, Li PC, Shi JH, Wang ZH, Cheng Y, He JX, Shi YK, Chen WQ, et al: OS outcomes to anlotinib in patients (pts) with refractory NSCLC of both wild-type (WT) and mutant EGFR. J Clin Oncol 36 (15 Suppl): e21013, 2018.
68. Shao L, Wang W, Song Z and Zhang Y: The efficacy and safety of anlotinib treatment for advanced lung cancer. Onco Targets Ther 12: 6549-6554, 2019.

69. Gao W, He J, Jin SD, Xu J, Yu TF, Wang W, Zhu Q, Dai H, Wu H, Liu YQ, et al: Association of initial epidermal growth factor receptor tyrosine kinase inhibitors treatment and EGFR exon 19 deletion with frequency of the T790M mutation in non-small cell lung cancer patients after resistance to first-line epidermal growth factor receptor tyrosine kinase inhibitors. Onco Targets Ther 12: 9495-9504, 2019.

70. Kwak EL, Sordella R, Bell DW, Godin-Heymann N, Okimoto RA, Brannigan BW, Harris PL, Driscoll DR, Fidias P, Lynch TJ, et al: Irreversible inhibitors of the EGF receptor may circumvent acquired resistance to gefitinib. Proc Natl Acad Sci USA 102: 7665-7670, 2005

71. Zhou M, Chen X, Zhang H, Xia L, Tong X, Zou L, Hao R, Pan J, Zhao X, Chen D, et al: China national medical products administration approval summary: Anlotinib for the treatment of advanced non-small cell lung cancer after two lines of chemotherapy. Cancer Commun (Lond) 39: 36, 2019.

72. Si X, Zhang L, Wang H, Zhang X, Wang M, Han B, Li K, Wang Q, Shi J, Wang Z, et al: Quality of life results from a randomized, double-blinded, placebo-controlled, multi-center phase III trial of anlotinib in patients with advanced non-small cell lung cancer. Lung Cancer 122: 32-37, 2018.

73. Liang W, Zhao Y, Zhang Y, Han X, Yang X, He J, Li K and Han B: P2.01-01 The impact of anlotinib on brain metastases of NSCLC: Post-hoc analysis of a phase III randomized control trial (ALTER0303). J Thorac Oncol 13 (10 Suppl): S665, 2018.

74. Wang J, Zhao Y, Wang Q, Zhang L, Shi J, Wang Z, Cheng Y, He J, Shi Y, Yu H, et al: Prognostic factors of refractory NSCLC patients receiving anlotinib hydrochloride as the third- or further-line treatment. Cancer Biol Med 15: 443-451, 2018.

75. Dobbin SJH, Cameron AC, Petrie MC, Jones RJ, Touyz RM and Lang NN: Toxicity of cancer therapy: What the cardiologist needs to know about angiogenesis inhibitors. Heart 104: 1995-2002, 2018.

76. Si X, Zhang L, Wang H,Zhang X, Wang M, Han B, Li K, Wang Q, Shi J, Wang Z, et al: Management of anlotinib-related adverse events in patients with advanced non-small cell lung cancer: Experiences in ALTER-0303. Thorac Cancer 10: 551-556, 2019.

77. Rimassa L, Danesi R, Pressiani T and Merle P: Management of adverse events associated with tyrosine kinase inhibitors: Improving outcomes for patients with hepatocellular carcinoma. Cancer Treat Rev 77: 20-28, 2019.

78. Walko CM and Grande C: Management of common adverse events in patients treated with sorafenib: Nurse and pharmacist perspective. Semin Oncol 41 (Suppl 2): S17-S28, 2014.

79. Miura S, Fujino M, Matsuo Y, Tanigawa H and Saku K: Nifedipine-induced vascular endothelial growth factor secretion from coronary smooth muscle cells promotes endothelial tube formation via the kinase insert domain-containing receptor/fetal liver kinase-1/NO pathway. Hypertens Res 28: 147-153, 2005.

80. Kim SY, Kim SM, Chang H, Kim BW, Lee YS, Chang HS and Park CS: Safety of tyrosine kinase inhibitors in patients with differentiated thyroid cancer: Real-world use of lenvatinib and Sorafenib in Korea. Front Endocrinol (Lausanne) 10: 384, 2019.

81. Nikolaou V, Syrigos K and Saif MW: Incidence and implications of chemotherapy related hand-foot syndrome. Expert Opin Drug Saf 15: 1625-1633, 2016

82. Srinivas S, Stein D, Teltsch DY, Tao S, Cisar L and Ramaswamy K: Real-world chart review study of adverse events management in patients taking tyrosine kinase inhibitors to treat metastatic renal cell carcinoma. J Oncol Pharm Pract 24: 574-583, 2018.

83. Cheville AL, Kollasch J, Vandenberg J, Shen T, Grothey A, Gamble G and Basford JR: A home-based exercise program to improve function, fatigue, and sleep quality in patients with stage IV lung and colorectal cancer: A randomized controlled trial. J Pain Symptom Manage 45: 811-821, 2013.

84. Rugo HS, Di Palma JA, Tripathy D, Bryce R, Moran S, Olek E and Bosserman L: The characterization, management, and future considerations for ErbB-family TKI-associated diarrhea. Breast Cancer Res Treat 175: 5-15, 2019.

85. McCole DF and Barrett KE: Decoding epithelial signals: Critical role for the epidermal growth factor receptor in controlling intestinal transport function. Acta Physiol (Oxf) 195: 149-159, 2009.

86. Bowen JM, Mayo BJ, Plews E, Bateman E, Stringer AM, Boyle FM, Finnie JW and Keefe DM: Development of a rat model of oral small molecule receptor tyrosine kinase inhibitor-induced diarrhea. Cancer Biol Ther 13: 1269-1275, 2012. 
87. Lu J, Shi Q, Zhang L, Wu J, Lou Y, Qian J, Zhang B, Wang S Wang H, Zhao X and Han B: Integrated transcriptome analysis reveals KLK5 and L1CAM predict response to anlotinib in NSCLC at 3rd line. Front Oncol 9: 886, 2019.

88. Liu Z, Wang J, Meng Z, Wang X, Zhang C, Qin T, Chen J, Jiang X, Wang L, Lin L, et al: CD31-labeled circulating endothelial cells as predictor in anlotinib-treated non-small-cell lung cancer: Analysis on ALTER-0303 study. Cancer Med: Jun 1, 2018 (Epub ahead of print).

89. Lu J, Zhang W, Yan B, Li H, Zhang L, Dong Y, Qian J, Wang S, Zhang B, Wu J, et al: Tumor mutation index as a biomarker for responsive stratification on multi-targeted TKI anlotinib: An ALTER-0303 companion diagnostic study. Ann Oncol 29: (Suppl 9): ix113, 2018.

90. Lu J, Zhong H, Wu J, Chu T, Zhang L, Li H, Wang Q, Li R, Zhao Y, Gu A, et al: Circulating DNA-based sequencing guided anlotinib therapy in non-small cell lung cancer. Adv Sci (Weinh) 6: 1900721, 2019.
91. Chen D, Xu J, Zhao Y, Chu T, Zhong H, Han B and Zhong R: Prognostic value of tumor cavitation in extensive-stage small-cell lung cancer patients treated with anlotinib. J Cancer Res Clin Oncol 146: 401-406, 2020.

92. Ohm JE and Carbone DP: VEGF as a mediator of tumor-associated immunodeficiency. Immunol Res 23: 263-272, 2001.

93. Garber K: Promising early results for immunotherapy-antiangiogenesis combination. J Natl Cancer Inst 106: pii: dju392, 2014.

94. Rizvi NA, Antonia SJ, Shepherd FA, Chow LQ, Goldman J, Shen Y, Chen AC and Gettinger S: Nivolumab (Anti-PD-1; BMS-936558, ONO-4538) maintenance as monotherapy or in combination with bevacizumab (BEV) for non-small cell lung cancer (NSCLC) previously treated with chemotherapy: Metastatic non-small cell lung cancer. Int J Radiat Oncol 90 (5 Suppl): S32, 2014.

95. Ahn MJ, Sun JM, Lee SH, Ahn JS and Park K: EGFR TKI combination with immunotherapy in non-small cell lung cancer. Expert Opin Drug Saf 16: 465-469, 2017. 